\title{
Visualizing resonances in finite volume
}

\author{
Peng Guo $\circledast^{1,2,3, *}$ and Bingwei Long $\oplus^{1, \dagger}$ \\ ${ }^{1}$ College of Physics, Sichuan University, Chengdu 610065, China \\ ${ }^{2}$ Department of Physics and Engineering, California State University, Bakersfield, California 93311, USA \\ ${ }^{3}$ Kavli Institute for Theoretical Physics, University of California, Santa Barbara, California 93106, USA
}

(Received 22 July 2020; accepted 8 October 2020; published 27 October 2020)

\begin{abstract}
In present work, we explore and experiment with an alternative approach to studying resonance properties in a finite volume. By analytic continuing the finite lattice size $L$ into a complex plane, the oscillating behavior of the finite volume Green's function is mapped onto an infinite volume Green's function that is corrected by exponentially decaying finite volume effect. The analytic continuation technique thus can be applied to study resonance properties directly in finite volume dynamical equations.
\end{abstract}

DOI: 10.1103/PhysRevD.102.074508

\section{INTRODUCTION}

The Dalitz plot is a powerful tool in particle physics to extract information from processes involving three-particle final states. For instance, the $u$ - and $d$-quark mass difference can be extracted by the Dalitz analysis of $\eta \rightarrow 3 \pi$ [1-7]. Since many resonances emerge in few-hadron systems, the Dalitz plot also plays an important role in the study of resonance dynamics from experimental data, e.g., the coupled-channel analysis of $\rho$ and $K^{*}$ resonances dynamics in $[8,9]$.

On the theory side, lattice quantum chromodynamics (LQCD) has been one of the promising ab initio methods to provide understanding of few-hadron dynamics from the Standard Model. In past few years, many progresses have been made in LQCD calculations towards understanding multihadron systems [10-27]. However, LQCD normally puts out discrete energy spectra of few-hadron systems, because of the finite volume inherent to the method, rather than reaction amplitudes, which are needed to generate the Dalitz plot. Lattice QCD calculations are usually performed with the spatial periodic boundary condition. In the twobody sector, connections between infinite-volume reaction amplitudes and energy levels in a cubic box under periodic boundary condition can be constructed in a compact and elegant equation, normally referred as the Lüscher formula [28], and it has since been extended to cases including moving frames and coupled channels [29-39].

\footnotetext{
pguo@csub.edu

’bingwei@scu.edu.cn
}

Published by the American Physical Society under the terms of the Creative Commons Attribution 4.0 International license. Further distribution of this work must maintain attribution to the author(s) and the published article's title, journal citation, and DOI. Funded by SCOAP .
Various approaches to finite-volume three-particle dynamics exist [40-69], such as the relativistic all-orders perturbation theory [45-49], the effective theory based approach [40-42,50,51,70], and the Faddeev type equation based variational approach [65-69]. As pointed out in Ref. [71], though the quantization conditions are formulated in different ways depending on a specific approach, all approaches share the same strategy and similar features. The infinite-volume reaction amplitudes are in fact not directly extracted from lattice data. Only subprocess interactions or associated subprocess amplitudes are obtained from the quantization condition, and the total infinite volume reaction amplitudes have to be computed in a separate step. Two-step procedures seem like a compromise solution, and one may hope to have an ultimate formalism that grants the user direct access to infinitevolume reaction amplitudes from the LQCD energy levels, independently of interhadron interaction models. But deriving such relations beyond two-body systems poses great challenges. With two-step procedures, the finitevolume and infinite-volume physics can be dealt separately, eventually linked by interhadron interactions at one's proposal. Therefore, the quantization condition is free of infinite-volume reaction amplitudes, and it is more straightforward to implement in the practical data analysis of the LQCD results. The model dependence of the interhadron potential can be assessed by how well it fits to the LQCD energy levels. However, scattering observables, such as the Dalitz plot, must be computed in a separate step.

In the present work, we aim to explore and experiment with an alternative approach by fully taking advantage of Faddeev type integral equations in a finite volume. By the analytic continuation of a box size $L$ into a complex plane, the mapping relation of a finite volume Green's function in different energy domains can be established as the consequence of global spatial symmetry. Therefore, in a finite 
volume, $L$ may be used as a tunable parameter to turn an oscillating finite volume Green's function into the infinite volume Green's function with some exponentially decaying finite volume corrections. Hence, the resonance shows up as a peak as well in finite volume scattering amplitudes, even for small $L$ values.

The paper is organized as follows. The analytic continuation technique is explained and demonstrated in Sec. II with the one-dimension case. We summarize the findings in Sec. III.

\section{ANALYTIC CONTINUATION OF FINITE VOLUME AMPLITUDES}

The finite-volume Green's function is qualitatively different from its infinite-volume counterpart in that one has poles in the complex energy plane corresponding to discrete levels, and the other has branch cuts corresponding to a continuous spectrum. So a very large cubic box must be used if one would like to approximate scattering states in finite volume. However, as we will show in this section, if $L$ is analytically continued to its complex plane, finitevolume amplitudes can resemble quite well the actual reaction amplitudes, even for relatively small values of $L$. To keep the technical discussion at a manageable level, our presentation in what follows will be only limited to a nonrelativistic few-body system in one-dimensional space. The physical application of such a technique, such as the Dalitz plot of $\eta, \eta^{\prime} \rightarrow 3 \pi$, will be presented in a separate work.

\section{A. Finite volume amplitude and Lüscher formula}

Let us start the discussion with two-body interactions in finite volume. One of the major tasks of investigating finitevolume dynamics is to look for the discrete eigenenergies of few-body systems in a periodic box. These energy eigenstates are stationary and are described by a homogeneous Lippmann-Schwinger (LS) equation in the twobody case,

$$
\phi_{L}(E)=G_{L}(E) V \phi_{L}(E)
$$

where $G_{L}$ and $\phi_{L}$ stand for the finite volume Green's function and wave function, respectively, and $V$ denotes the interaction potential of particles. Equivalently, the homogeneous LS equation can be rewritten as

$$
t_{L}(E)=V G_{L}(E) t_{L}(E),
$$

where

$$
t_{L}(E)=-V \phi_{L}(E)
$$

The energies of stationary states are those letting the following determinant vanish:

$$
\operatorname{det}\left[I-V G_{L}(E)\right]=0, \quad E \in\left\{E_{1}, \ldots, E_{n}, \ldots\right\} .
$$

It is useful to introduce an operator $\tau_{L}(E)$ that satisfies the inhomogeneous LS equation,

$$
\tau_{L}(E)=-V+V G_{L}(E) \tau_{L}(E) .
$$

The solution of Eq. (4) is symbolically given by

$$
\tau_{L}(E)=-\frac{1}{\frac{1}{V}-G_{L}(E)},
$$

which will be used in the three-body finite-volume LS equations. The poles of the $\tau_{L}(E)$ amplitude also yield eigenenergy solutions of a stationary state of the few-body finite volume system, which is consistent with the quantization condition given by Eq. (3).

The matrix element of $\tau_{L}(E)$ between two plane waves defines the finite-volume transition amplitude, which could be on shell or off shell. Using 1D as the example, the off shell finite-volume amplitude in the plane wave basis is given by

$$
\tau_{L}\left(k ; E ; k^{\prime}\right)=\left\langle k\left|\tau_{L}(E)\right| k^{\prime}\right\rangle,
$$

where

$$
\left(k, k^{\prime}\right) \in \frac{2 \pi n}{L}, \quad \text { and } \quad n \in \mathcal{Z} .
$$

Equation (4) thus yields

$$
\begin{aligned}
\tau_{L}\left(k ; E ; k^{\prime}\right)= & -\tilde{V}\left(k-k^{\prime}\right) \\
& +\sum_{p} \tilde{V}(k-p) \tilde{G}_{L}(p ; E) \tau_{L}\left(p ; E ; k^{\prime}\right),
\end{aligned}
$$

where the two-body finite-volume Green's function in the center of mass $(\mathrm{CM})$ frame is given by

$$
\tilde{G}_{L}(p ; E)=\frac{1}{L} \frac{1}{m E-p^{2}} .
$$

Equation (8) resembles the LS equation in an infinite volume for scattering states, hence discrete $\left(k^{\prime}, k\right) \neq \sqrt{m E}$ may be interpreted as off shell incoming and outgoing momenta, respectively.

Considering the short-range interaction approximation, $\tilde{V}\left(k-k^{\prime}\right) \simeq \tilde{V}(0)=V$, the solution to Eq. (8) is thus dominated primarily by diagonal terms of off shell amplitudes, $\tau_{L}(k ; E ; k) \simeq \tau_{L}(E)$, which are normally also referred to as on shell approximation; see Refs. [31,72]. Hence, Eq. (8) is reduced to a algebra equation, and the solution is given by 


$$
\tau_{L}(E)=-\frac{1}{\frac{1}{V}-G_{L}(0, E)}
$$

where

$$
G_{L}(0, E)=\frac{1}{L} \sum_{p=\frac{2 \pi n}{L}, n \in \mathbb{Z}} \frac{1}{m E-p^{2}}=\frac{\cot \frac{\sqrt{m E}}{2} L}{2 \sqrt{m E}} .
$$

The potential term $\frac{1}{V}$ is related to the scattering phase shift and infinite volume Green's function,

$$
\frac{1}{V}=-\frac{1}{2 \sqrt{m E}}[\cot \delta(E)-i]+G_{\infty}(0, E),
$$

where

$$
G_{\infty}(0, E)=\int \frac{d p}{2 \pi} \frac{1}{m E-p^{2}}=-\frac{i}{2 \sqrt{m E}} .
$$

Thus, Eq. (10) can be rewritten in the form that is associated to the Lüscher formula,

$$
\tau_{L}(E)=\frac{1}{2 \sqrt{m E}} \frac{1}{\cot \delta(E)-\mathcal{M}_{L}(E)},
$$

where $\mathcal{M}_{L}(E)$ is Lüscher's zeta function,

$$
\mathcal{M}_{L}(E)=i+2 \sqrt{m E}\left[G_{\infty}(0, E)-G_{L}(0, E)\right] .
$$

The pole of $\tau_{L}(E)$ yields the Lüscher formula,

$$
\cot \delta(E)-\mathcal{M}_{L}(E)=0 .
$$

Although the expression of $\tau_{L}(E)$ in Eq. (10) has a similar structure as its infinite volume counterpart, the on shell two-body scattering amplitude,

$$
\tau_{\infty}(E)=-\frac{1}{\frac{1}{V}-G_{\infty}(0, E)}=\frac{1}{2 \sqrt{m E}} \frac{1}{\cot \delta(E)-i},
$$

$\tau_{L}(E)$ and $\tau_{\infty}(E)$ behave significantly different due to superficially divergent analytic appearance of finite volume and infinite volume Green's functions. In finite volume, $G_{L}(0, E)$ is a periodic oscillating real function, compared with the infinite volume counterpart $G_{\infty}(0, E)$, which is purely imaginary. The difference between $G_{L}(0, E)$ and $G_{\infty}(0, E)$ may be understood from the analytic properties of Green's functions; in infinite volume, Green's function is determined by the branch cut lying on positive real axis,

$$
G_{\infty}(0, E)=\frac{1}{2 \pi} \int_{0}^{\infty} d s^{\prime} \frac{\sqrt{\frac{1}{s^{\prime}}}}{m E-s^{\prime}} .
$$

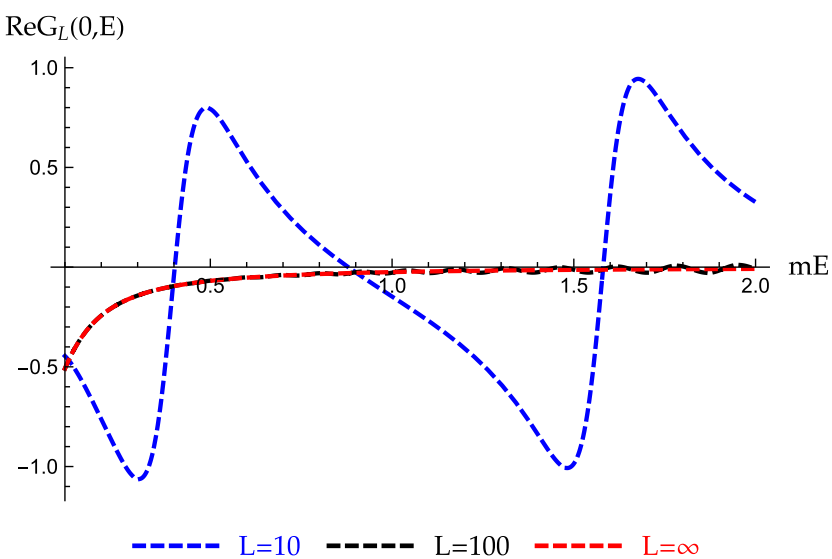

$\operatorname{ImG}_{L}(0, \mathrm{E})$

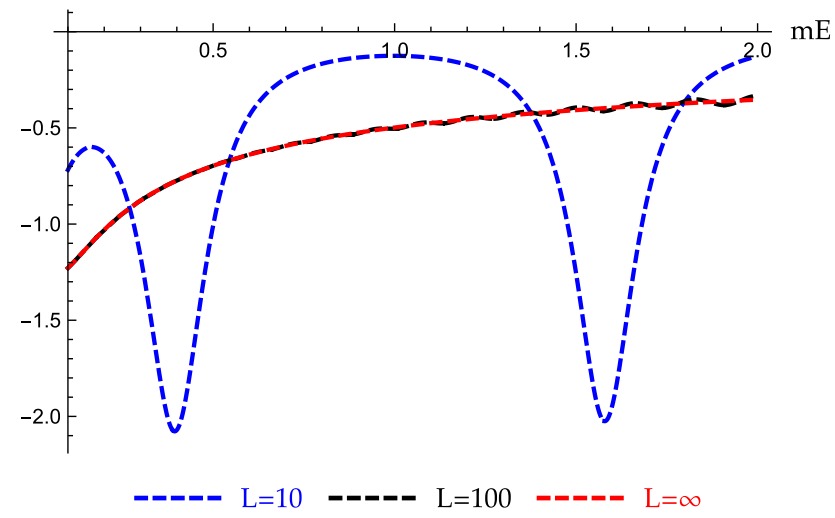

FIG. 1. The comparison of $G_{L}(0, E)$ and $G_{\infty}(0, E)$ with the complex argument $m E+i \epsilon$, where $\epsilon=0.1$ and $L=10$ (blue), 100 (black), and $\infty$ (red).

For the values of $m E$ taken slightly above the real axis by $m E+i \epsilon$, the principle part of the above interaction vanishes; only the absorptive part survives and that yields the imaginary part $-\frac{i}{2 \sqrt{m E}}$. However, in finite volume, the branch cut dissolves into discrete poles lying on the real axis; therefore, for the values of $m E$ not overlapping with pole positions, only the principle part contributes. It is also interesting to see that for finite $i \epsilon$, both $G_{L}(0, E)$ and $G_{\infty}(0, E)$ becomes complex, and the sharp oscillating behavior of $G_{L}(0, E)$ is smoothed out and starts matching with $G_{\infty}(0, E)$ when $L \gg 1 / \sqrt{\epsilon}$; see Fig. 1 as a example. In other words, as $\epsilon \rightarrow 0, \tau_{L}(E)$ and $\tau_{\infty}(E)$ indeed behaves significantly different for finite $L$, and $\tau_{L}(E) \rightarrow \tau_{\infty}(E)$ only when $L \rightarrow \infty$. Therefore, $\tau_{L}(E)$ for finite $L$ normally are not considered as a useful tool for the identification of a sharp resonance that on the contrary appears as a peak in $\tau_{\infty}(E)$.

Next, we will explain how the analytic continuation technique may allow one to have finite volume amplitude that resembles the behavior of an infinite volume amplitude for even finite $L$ and real $m E$ values with $\epsilon \rightarrow 0$. It turns out that the analytic continuation technique is the direct consequence of the global symmetry of Green's function in complex space. 


\section{B. Global symmetry and analytic continuation of Green's function}

Using again a 1D nonrelativistic few-body system as an example, in infinite volume, a two-body Green's function satisfies

$$
\left(m E+\frac{d^{2}}{d x^{2}}\right) G_{\infty}(x, E)=\delta(x)
$$

where the physical value of the position $x$ is defined on a real axis. Now, let us extend $x$ to the complex plane by multiplying a phase factor $e^{i \theta}$,

$$
x \rightarrow x e^{i \theta},
$$

where $\theta \in[0, \pi]$. We remark that the method of extension of $x$ to complex plane is also known as the complex scaling method in nuclear and atomic physics; see Refs. [73-75]. In complex space, Eq. (19) yields

$$
\left(m E e^{i 2 \theta}+\frac{d^{2}}{d x^{2}}\right)\left[e^{-i \theta} G_{\infty}\left(x e^{i \theta}, E\right)\right]=\delta(x) .
$$

One can conclude that the Green's function equation is invariant under the global rotation of space in a complex plane, and $e^{-i \theta} G_{\infty}\left(x e^{i \theta}, E\right)$ is related to the solution of Eq. (19) on a real axis with the eigenenergy of $m E e^{i 2 \theta}$; hence,

$$
G_{\infty}\left(x e^{i \theta}, E\right)=e^{i \theta} G_{\infty}\left(x, E e^{i 2 \theta}\right) .
$$

For $\theta=\frac{\pi}{2}$; thus,

$$
G_{\infty}(0, E)=i G_{\infty}(0,-E) .
$$

This is indeed consistent with the analytic expression in Eq. (13). That is to say that the Green's function in a physical region $(E>0)$ is mirrored to an unphysical region $(E<0)$ by Eq. (22) as the consequence of global spatial symmetry. In infinite volume, $E$ is the only tunable parameter in $G_{\infty}(0, E)$ that can be used to cross from physical region to unphysical region or vice versa.

The finite volume two-body Green's function is given by

$$
\left(m E+\frac{d^{2}}{d x^{2}}\right) G_{L}(x, E)=\sum_{n \in \mathcal{Z}} \delta(x+n L) .
$$

Similarly, we want to extend the finite system to the complex plane by a global spatial rotation, $x \rightarrow x e^{i \theta}$ and also $L \rightarrow L e^{i \theta}$; thus, we find

$$
\left(m E e^{i 2 \theta}+\frac{d^{2}}{d x^{2}}\right)\left[e^{-i \theta} G_{L e^{i \theta}}\left(x e^{i \theta}, E\right)\right]=\sum_{n \in \mathcal{Z}} \delta(x+n L) .
$$

Equation (24) yields a useful relation,

$$
G_{L e^{i \theta}}(0, E)=e^{i \theta} G_{L}\left(0, E e^{i 2 \theta}\right) .
$$

A key observation is that because of the extra tunable parameter $L$ in a finite volume, now, Eq. (25) allows one to navigate freely between the physical and unphysical regions of a finite volume Green's function with a fixed value of $E$.

It is also worth noting that though $G_{L}(0, E)$ and $G_{\infty}(0, E)$ are significantly different for $E>0$ with finite $L$, below the physical threshold $(E<0)$,

$$
G_{L}(0, E<0)=-\frac{\operatorname{coth} \frac{\sqrt{|m E|} L}{2}}{2 \sqrt{|m E|}},
$$

quickly approaches its infinite volume counterpart $G_{\infty}(0, E<0)=-\frac{1}{2 \sqrt{|m E|}}$ as increasing $L$, due to the fact that

$\operatorname{coth} \frac{\sqrt{m E}}{2} L=1+2 e^{-\sqrt{m E} L}+2 e^{-2 \sqrt{m E} L}+\cdots \stackrel{L \rightarrow \infty}{\longrightarrow} 1$.

Now, using relation in Eq. (25), simply rotating $L \rightarrow i L$, we find

$G_{i L}(0, E)=-i \frac{\operatorname{coth} \frac{\sqrt{m E}}{2} L}{2 \sqrt{m E}}=G_{\infty}(0, E) \operatorname{coth} \frac{\sqrt{m E}}{2} L$.

Therefore, in the physical region $E>0, G_{i L}(0, E)$ behaves just as $G_{\infty}(0, E)$ with exponential decaying corrections due to the finite volume effect; see Fig. 2.

\section{Resonance in $i L$ space}

With the continuation $L \rightarrow i L$, the finite-volume on shell amplitude,

$$
\tau_{i L}(E)=\frac{1}{2 \sqrt{m E}} \frac{1}{\cot \delta(E)-\mathcal{M}_{i L}(E)},
$$

approximates very well $\tau_{\infty}(E)$ in the physical region of $E$, differing only by powers of $e^{-\sqrt{m E} L}$,

$\mathcal{M}_{i L}(E)=i\left(1+2 e^{-\sqrt{m E} L}+2 e^{-2 \sqrt{m E} L}+\cdots\right) \stackrel{L \rightarrow \infty}{\longrightarrow} i$.

We will illustrate how this helps identify resonances as a peak of $\tau_{i L}(E)$, just as in the infinite-volume case.

We propose a resonance model by replacing $V$ by

$$
m V_{0}+\frac{g_{\rho}}{m\left(E-m_{\rho}\right)},
$$



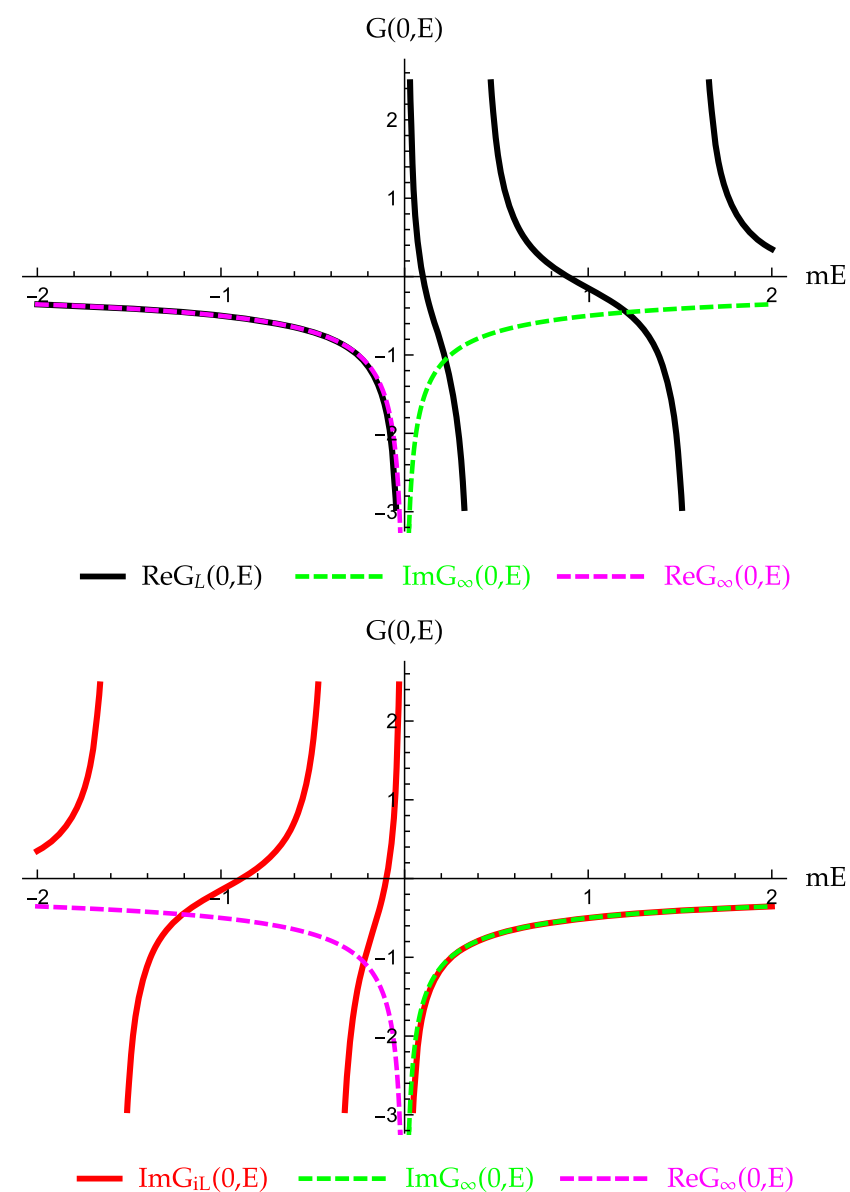

FIG. 2. Plot of $\operatorname{Re} G_{L}(0, E)$ (black solid) and $\operatorname{Im} G_{i L}(0, E)$ (red solid) with $L=10$ vs $\operatorname{Im} G_{\infty}(0, E)$ (dashed green) and $\operatorname{Re} G_{\infty}(0, E)$ (dashed magenta).

where $g_{\rho}$ and $m_{\rho}$ are the coupling constant and mass of the resonance, respectively, and $m V_{0}$ can be used to parametrize the background contribution. The infinite-volume scattering amplitude is thus given by

$$
\tau_{\infty}^{(\text {res })}(E)=-\frac{1}{\frac{1}{m V_{0}+\frac{g_{\rho}}{m\left(E-m_{\rho}\right)}}+\frac{i}{2 \sqrt{m E}}},
$$

to be compared with the analytically continued finitevolume amplitude,

$$
\tau_{i L}^{(\text {res })}(E)=-\frac{1}{\frac{1}{m V_{0}+\frac{g \rho}{m\left(E-m_{\rho}\right)}}+\frac{i}{2 \sqrt{m E}} \operatorname{coth} \frac{\sqrt{m E}}{2} L} .
$$

The comparison of $\tau_{\infty}^{(\mathrm{res})}(E)$ and $\tau_{i L}^{\text {(res) }}(E)$ for a resonance model is plotted in Fig. 3.

In infinite volume, the resonance pole position is given by

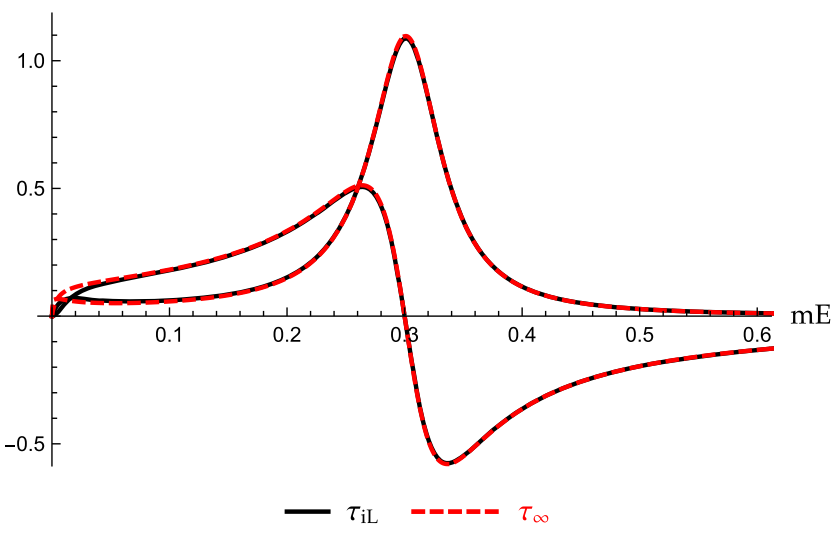

FIG. 3. The comparison of $\tau_{i L}^{(\text {res }, 0)}(0, E)$ (solid black) and $\tau_{\infty}^{(\text {res, } 0)}(0, E)$ (dashed red) with $L=10, m V_{0}=0, g_{\rho}=0.04$, and $m m_{\rho}=0.3$.

$$
\frac{1}{m V_{0}+\frac{g_{\rho}}{m\left(E-m_{\rho}\right)}}+\frac{i}{2 \sqrt{m E}}=0
$$

and in finite volume, the pole position of $\tau_{i L}^{(\text {res, }, 0)}(E)$ is shifted,

$$
\frac{1}{m V_{0}+\frac{g_{\rho}}{m\left(E-m_{\rho}\right)}}+i \frac{\operatorname{coth} \frac{\sqrt{m E}}{2} L}{2 \sqrt{m E}}=0
$$

Figure 4 depicts the resonance pole of $\tau_{i L}^{(\text {res, }, 0)}(E)$ for various values of $L$ and the pole of $\tau_{\infty}^{\text {(res) }}(E)$. It shows clearly how rapidly the finite-volume pole approaches its infinitevolume limit.

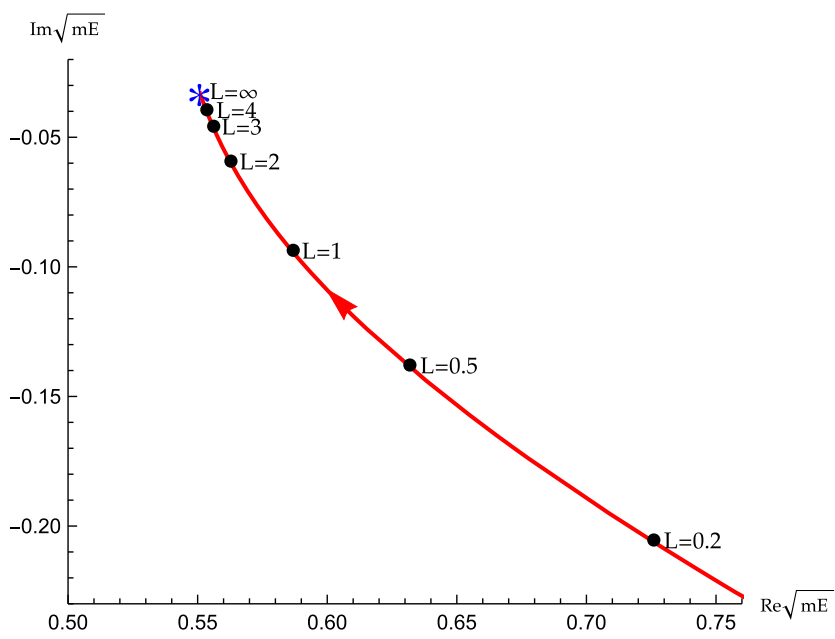

FIG. 4. The trajectory of the resonance pole position as the function of $L$ with the parameters of a resonance model: $m V_{0}=0, g_{\rho}=0.04$, and $m m_{\rho}=0.3$. An arrow indicates increasing $L$ direction. 


\section{Inhomogeneous three-particle Faddeev type equation in finite volume}

The idea presented in previous sections can now be applied into three-body systems. In finite volume, the stationary solutions of a three-body system may be described by an homogeneous Schrödinger equation,

$$
\Phi_{L}=G_{L}(E)\left(V_{12}+V_{23}+V_{31}\right) \Phi_{L},
$$

where the subscript of $V_{\alpha \beta}$ is used to describe the interaction among different pairs. The Eq. (35) can be converted into homogeneous Faddeev type coupled dynamical equations; see [66-69]. In a simple case with three identical particles interacting through only a pairwise interaction, only one dynamical equation is required; symbolically, it is given by

$$
T_{L}(E)=\frac{2}{\frac{1}{V}-G_{L}(E)} G_{L}(E) T_{L}(E)
$$

where $T_{L}(E)=-V \Phi_{L}(E)$ stands for the finite volume Faddeev three-body amplitude. Homogeneous Eqs. (36) or (35) thus yield the quantization condition,

$$
\operatorname{det}\left[I-\frac{2}{\frac{1}{V}-G_{L}(E)} G_{L}(E)\right]=0,
$$

which determines the discrete eigenenergy of stationary solutions that satisfies the periodic boundary condition in a finite volume. In addition to establishing the quantization condition and obtaining eigenenergies, the finite volume wave function may also be computed from the dynamical equations, Eqs. (36) and (35); the technical details of extracting a few-body finite volume wave function are presented in Appendix A.

To compare with infinite volume scattering amplitudes, let us introduce a three-body operator $\mathcal{T}_{L}(E)$ that satisfies the inhomogeneous three-body equation,

$\mathcal{T}_{L}(E)=-\frac{1}{\frac{1}{V}-G_{L}(E)}+\frac{2}{\frac{1}{V}-G_{L}(E)} G_{L}(E) \mathcal{T}_{L}(E)$

the poles of $\mathcal{T}_{L}(E)$ correspond to the stationary solutions that are also described by the homogeneous equation, Eq. (36). The off shell finite volume amplitude in a plane wave basis that resembles the scattering amplitude in infinite volume may be introduced by

$$
\mathcal{T}_{L}\left(k_{1}, k_{2} ; E ; k_{1}^{\prime}, k_{2}^{\prime}\right)=\left\langle k_{1}, k_{2}\left|\mathcal{T}_{L}(E)\right| k_{1}^{\prime} k_{2}^{\prime}\right\rangle_{L},
$$

where $\left(k_{1}, k_{2}\right)$ and $\left(k_{1}^{\prime}, k_{2}^{\prime}\right) \in \frac{2 \pi}{L} n, n \in \mathbb{Z}$ represent outgoing and incoming two independent momenta of particles. While considering only the pairwise contact interaction, $\mathcal{T}_{L}\left(k_{1}, k_{2} ; E ; k_{1}^{\prime}, k_{2}^{\prime}\right)$ depends only on a single outgoing momentum, e.g., in a (13) channel,

$$
\begin{aligned}
\mathcal{T}_{L}\left(k_{1}, k_{2} ; E ; k_{1}^{\prime}, k_{2}^{\prime}\right)= & -\int_{L} d r_{13} d r_{23} e^{-i k_{1} r_{13}} e^{-i k_{2} r_{23}} \\
& \times m V_{0} \delta\left(r_{13}\right) \phi_{L}\left(r_{13}, r_{23} ; k_{1}^{\prime}, k_{2}^{\prime}\right) \\
= & \mathcal{T}_{L}\left(k_{2} ; E ; k_{1}^{\prime}, k_{2}^{\prime}\right) .
\end{aligned}
$$

The off shell inhomogeneous three-body LS equation for a pairwise contact interaction is thus given in a compact form,

$$
\begin{aligned}
\mathcal{T}_{L} & \left(k ; E ; k_{1}^{\prime}, k_{2}^{\prime}\right) \\
= & \tau_{L}^{(k)}(E)\left\langle k \mid k_{1}^{\prime} k_{2}^{\prime}\right\rangle_{L} \\
& -2 \tau_{L}^{(k)}(E) \sum_{p=\frac{2 \pi n}{L}, n \in \mathbb{Z}} L \tilde{G}_{L}(k, p ; E) \mathcal{T}_{L}\left(p ; E ; k_{1}^{\prime}, k_{2}^{\prime}\right),
\end{aligned}
$$

where

$$
\left\langle k \mid k_{1}^{\prime} k_{2}^{\prime}\right\rangle_{L}=L\left[\delta_{k, k_{2}^{\prime}}+\delta_{k, k_{1}^{\prime}}+\delta_{k, k_{3}^{\prime}}\right]
$$

The three-body finite volume Green's function is given by

$$
\tilde{G}_{L}\left(p_{1}, p_{2} ; E\right)=\frac{2}{L^{2}} \frac{1}{2 m E-\sum_{i=1}^{3} p_{i}^{2}},
$$

and the two-body amplitude $\tau_{L}^{(k)}(E)$ in a moving frame is defined by

$$
\begin{aligned}
\tau_{L}^{(k)}(E) & =-\frac{1}{\frac{1}{m V_{0}}-\sum_{p} L \tilde{G}_{L}(k, p ; E)} \\
& =-\frac{1}{\frac{1}{m V_{0}}-\frac{\cot \frac{\sqrt{m E-\frac{3}{4} k^{2}}-\frac{k}{2}}{2} L+\cot \frac{\sqrt{m E-\frac{3}{4} k^{2}}+\frac{k}{2}}{2} L}{4 \sqrt{m E-\frac{3}{4} k^{2}}}} .
\end{aligned}
$$

The $k$ and $\sqrt{m E-\frac{3}{4} k^{2}}$ in $\tau_{L}^{(k)}(E)$ represent the total and the relative momenta of the two-particle pair, respectively.

Instead of solving the off shell LS equation, Eq. (41), it may be more convenient to introduce a half off shell amplitude that only depends on the outgoing momenta of particles by the sum over all the initial momenta $\left(k_{1}^{\prime}, k_{2}^{\prime}\right)$,

$$
\mathcal{T}_{L}(k ; E)=\frac{1}{\sum_{k_{1}^{\prime}, k_{2}^{\prime}}\left\langle k \mid k_{1}^{\prime} k_{2}^{\prime}\right\rangle_{L}} \sum_{k_{1}^{\prime}, k_{2}^{\prime}} \mathcal{T}_{L}\left(k ; E ; k_{1}^{\prime}, k_{2}^{\prime}\right)
$$

thus, Eq. (41) is converted into

$\mathcal{T}_{L}(k ; E)=\tau_{L}^{(k)}(E)-2 \tau_{L}^{(k)}(E) \sum_{p=\frac{2 \pi n}{L}, n \in \mathbb{Z}} L \tilde{G}_{L}(k, p ; E) \mathcal{T}_{L}(p ; E)$

The $\mathcal{T}_{L}(k ; E)$ amplitude may be used to describe the decay process. Equation (46) thus resembles an isobar model in 
the dispersion approach $[1,3,5,6,76]$, where $\tau_{L}^{(k)}(E)$ may be interpreted as a naive isobar pair term, and the second term in Eq. (46) thus corresponds to the isobar corrections due to the rescattering effect among different isobar pairs. Just as a two-body finite volume amplitude, for a finite value of $L$ and real $E, \mathcal{T}_{L}(k ; E)$ is a real and oscillating function that may not be in the suitable form for the task of the identification of resonance.

\section{E. Analytic continuation of three-particle Faddeev type equation in finite volume and solutions in $i L$ space}

The inhomogeneous three-body dynamical equation, Eq. (46), can be analytically continued into $i L$ space as well,

$$
\begin{aligned}
& \mathcal{T}_{i L}(k ; E) \\
& =\tau_{i L}^{(k)}(E)-2 \tau_{i L}^{(k)}(E) \sum_{p=\frac{2 \pi n}{i L}, n \in \mathbb{Z}}(i L) \tilde{G}_{i L}(k, p ; E) \mathcal{T}_{i L}(p ; E),
\end{aligned}
$$

where

$$
\tilde{G}_{i L}\left(p_{1}, p_{2} ; E\right)=\frac{2}{(i L)^{2}} \frac{1}{2 m E-\sum_{i=1}^{3} p_{i}^{2}},
$$

and

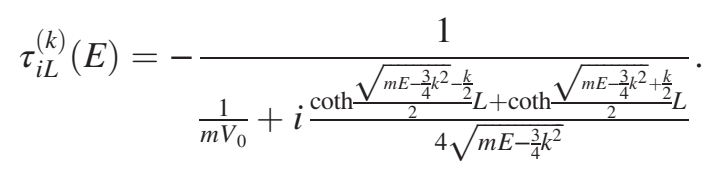

Now, it will be illustrated in what follows that the solution of Eq. (47), $\mathcal{T}_{i L}(k ; E)$, will behave similar to its counterpart in infinite volume, $\mathcal{T}_{\infty}(k ; E)$, which is given by

$$
\begin{aligned}
& \mathcal{T}_{\infty}(k ; E) \\
& \quad=\tau_{\infty}^{(k)}(E)-2 \tau_{\infty}^{(k)}(E) \int d p(2 \pi) \tilde{G}_{\infty}(k, p ; E) \mathcal{T}_{\infty}(p ; E),
\end{aligned}
$$

where

$$
\tilde{G}_{\infty}\left(p_{1}, p_{2} ; E\right)=\frac{2}{(2 \pi)^{2}} \frac{1}{2 m E-\sum_{i=1}^{3} p_{i}^{2}},
$$

and

$$
\tau_{\infty}^{(k)}(E)=-\frac{1}{\frac{1}{m V_{0}}+\frac{i}{2 \sqrt{m E-\frac{3}{4} k^{2}}} .}
$$

In addition to finding solutions of Eq. (47) in $i L$ space for discrete momenta values, Eq. (47) also allows us to analytically continue the argument $k$ in $\mathcal{T}_{i L}(k ; E)$ into real continuous values that can be used to compute a Dalitz plot etc.

First of all, Eq. (47) can be solved for $k \in \frac{2 \pi n}{i L}, n \in \mathbb{Z}$ by matrix inversion,

$$
\mathcal{T}_{i L}(p ; E)=\sum_{k}\left[\mathcal{D}_{i L}^{-1}(E)\right]_{p, k} \tau_{i L}^{(k)}(E)
$$

where $(k, p) \in \frac{2 \pi n}{i L}, n \in \mathbb{Z}$, and $\mathcal{D}_{i L}(E)$ matrix are given by

$$
\left[\mathcal{D}_{i L}(E)\right]_{p, k}=\delta_{p, k}+2 \tau_{i L}^{(k)}(E)(i L) \tilde{G}_{i L}(k, p ; E) .
$$

Next, plugging Eq. (53) into Eq. (47), therefore, the $\mathcal{T}_{i L}(k ; E)$ with a real continuous $k$ argument is now obtained by

$$
\mathcal{T}_{i L}(k ; E)=\tau_{i L}^{(k)}(E) g_{i L}(k ; E),
$$

where
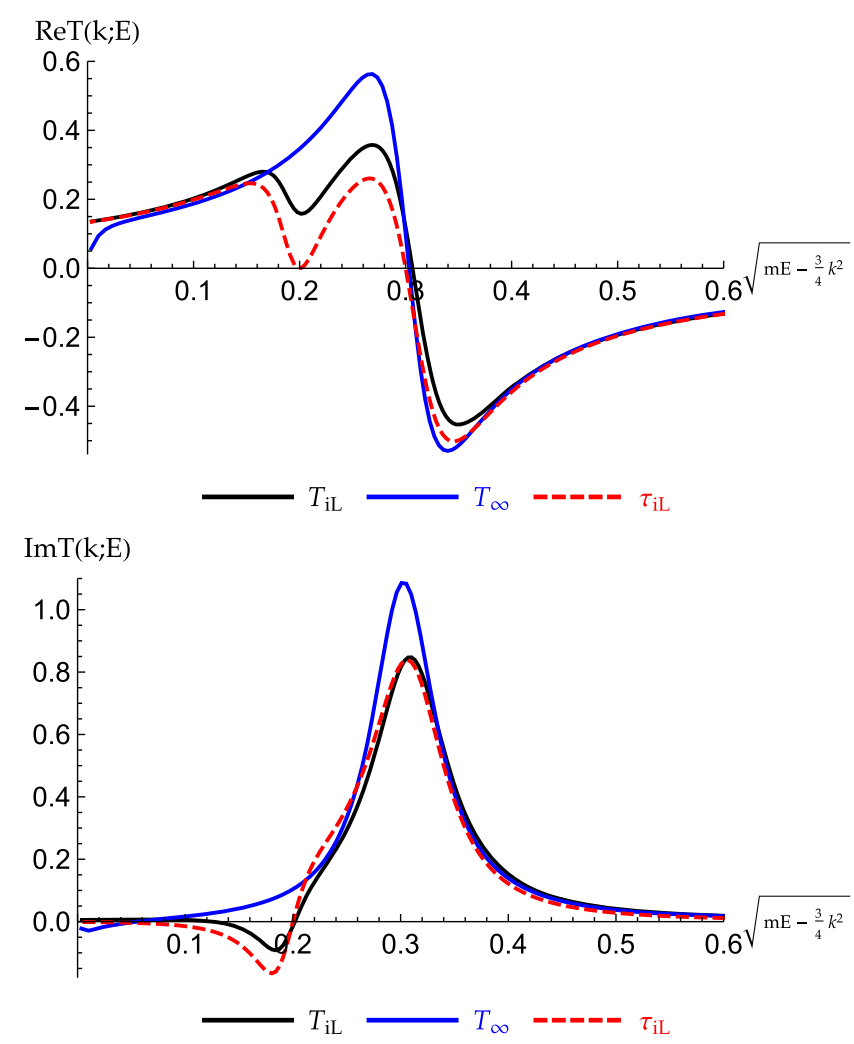

FIG. 5. The comparison of $\mathcal{T}_{i L}(k ; E)$ (solid black), $\tau_{i L}^{(\text {res }, k)}(E)$ (solid blue), and $\mathcal{T}_{\infty}(k ; E)$ with fixed $m E=0.8, L=10$, $m V_{0}=0, g_{\rho}=0.04$, and $m_{\rho}=0.3$. 

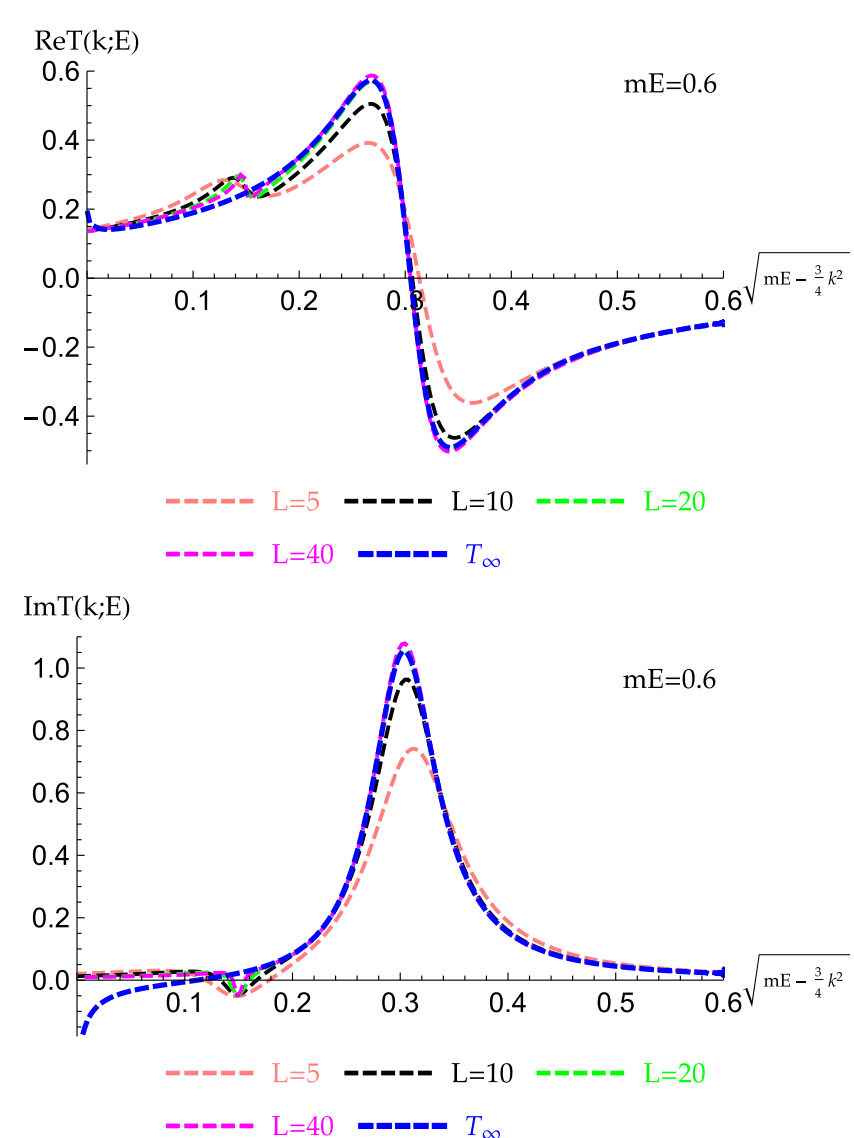

FIG. 6. The comparison of $\mathcal{T}_{i L}(k ; E)$ and $\mathcal{T}_{\infty}(k ; E)$ (blue) with multiple $L$ 's: $L=5$ (pink), 10 (black), 20 (green), 40 (magenta). Model parameters are fixed with $m E=0.6, m V_{0}=0, g_{\rho}=0.04$, and $m_{\rho}=0.3$.

$$
\begin{aligned}
& g_{i L}(k ; E) \\
& =1-2 \sum_{\left(p, p^{\prime}\right)=\frac{2 \pi n}{i L}, n \in \mathbb{Z}}(i L) \tilde{G}_{i L}(k, p ; E)\left[\mathcal{D}_{i L}^{-1}(E)\right]_{p, p^{\prime}} \tau_{i L}^{\left(p^{\prime}\right)}(E) .
\end{aligned}
$$

The second term in the $g_{i L}$ function describes the correction to the isobar model from crossed channels due to the rescattering effect.

Again, with a simple resonance model of a two-body amplitude by replacement $m V_{0} \rightarrow m V_{0}+\frac{g_{\rho}}{m E-\frac{3}{4} k^{2}-m m_{\rho}}$, and $\tau^{(k)}(E) \rightarrow \tau^{(\text {res }, k)}(E)$ in both finite and infinite volumes, for instance,

$$
\begin{aligned}
{\left[\tau_{i L}^{(\mathrm{res}, k)}(E)\right]^{-1}=} & -\frac{1}{m V_{0}+\frac{g_{\rho}}{m E-\frac{3}{4} k^{2}-m m_{\rho}}} \\
& -i \frac{\operatorname{coth} \frac{\sqrt{m E-\frac{3}{4} k^{2}}-\frac{k}{2}}{2} L+\operatorname{coth} \frac{\sqrt{m E-\frac{3}{4} k^{2}}+\frac{k}{2}}{2} L}{4 \sqrt{m E-\frac{3}{4} k^{2}}}
\end{aligned}
$$

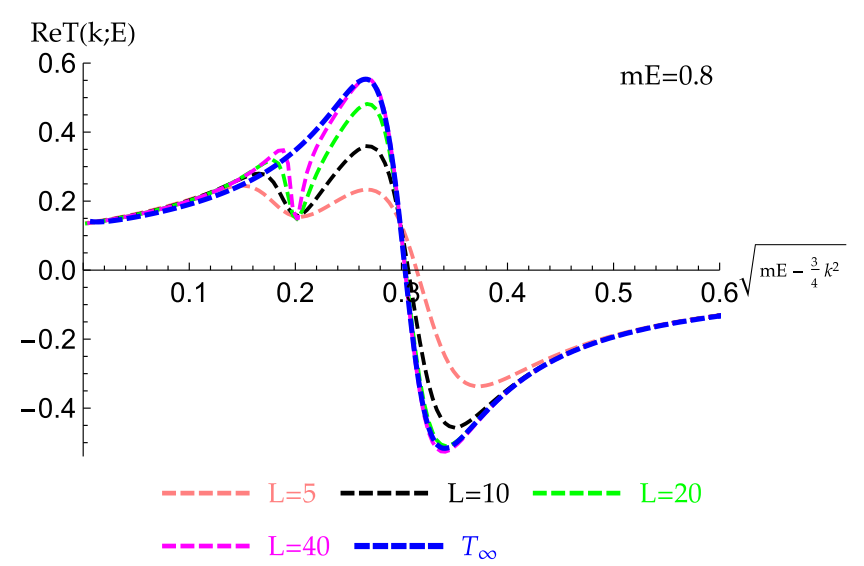

$\operatorname{ImT}(k ; E)$

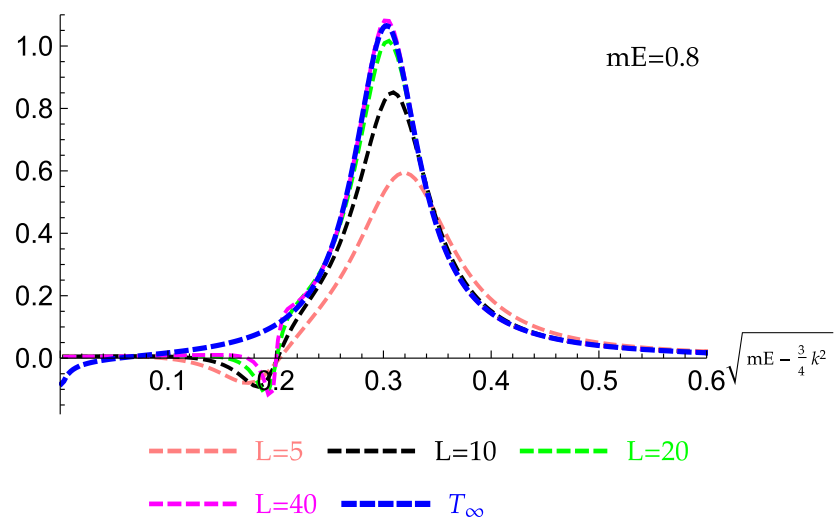

FIG. 7. The comparison of $\mathcal{T}_{i L}(k ; E)$ and $\mathcal{T}_{\infty}(k ; E)$ (blue) with a fixed $m E=0.8$ and multiple $L$ 's: $L=5$ (pink), 10 (black), 20 (green), 40 (magenta).

the comparison of numerical solutions of $\mathcal{T}_{i L}(k ; E)$ given by Eq. (55), and its counterpart in infinite volume, $\mathcal{T}_{\infty}(k ; E)$, given by Eq. (50), is shown in Fig. 5.

We remark that the cusp effect in $\mathcal{T}_{i L}(k ; E)$ for a real $k$ is a pure finite volume artifact; also see Figs. 6-8 for the plot of $\mathcal{T}_{i L}(k ; E)$ with multiple $L$ 's and $m E$ 's. The finite volume cusp originates from the analytic continuation of the finite volume three-body Green's function with real arguments,

$$
\begin{aligned}
& \sum_{p=\frac{2 \pi n}{i L}, n \in \mathbb{Z}}(i L) \tilde{G}_{i L}(k, p ; E) \\
& =\frac{1}{i L} \frac{1}{m E-k^{2}}+\frac{1}{i L} \sum_{p=\frac{2 \pi n}{i L}, n \neq 0} \frac{1}{m E-p^{2}-p k-k^{2}}
\end{aligned}
$$

Now, we can clearly see the pole term in a finite volume Green's function, $\frac{1}{m E-k^{2}}$, which appears to cause trouble for the real $k$ values near $\pm \sqrt{m E}$. In fact, this singular term shows up in $g_{i L}(k ; E)$ that is defined in Eq. (56) as a pole singularity; however, it also shows up in

$$
\tau_{i L}^{(k)}(E)=-\frac{1}{\frac{1}{m V_{0}}-\sum_{p=\frac{2 \pi n}{i L}}^{n \in \mathbb{Z}}(i L) \tilde{G}_{i L}(k, p ; E)} \propto\left(m E-k^{2}\right)
$$



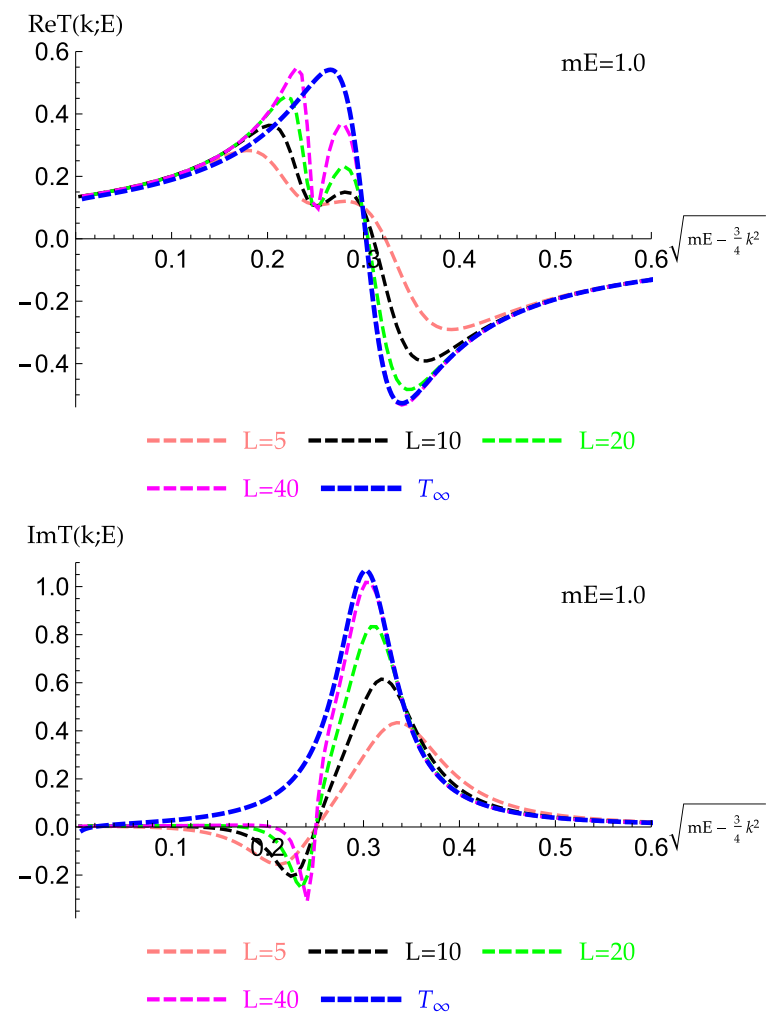

FIG. 8. The comparison of $\mathcal{T}_{i L}(k ; E)$ and $\mathcal{T}_{\infty}(k ; E)$ (blue) with a fixed $m E=0.8$ and multiple $L$ 's: $L=5$ (pink), 10 (black), 20 (green), 40 (magenta).

as zero, see the red dashed curves in Fig. 5. Therefore, the finite volume three-body amplitude $\mathcal{T}_{i L}(k ; E)=$ $\tau_{i L}^{(k)}(E) g_{i L}(k ; E)$ is free of a singularity in the end. Although the singularity is canceled out, the finite volume effect still shows up as a cusp, which is absent in infinite volume amplitudes. We also notice that the finite volume cusp is located at $k \sim \pm \sqrt{m E}$, while the location of a twobody resonance is around $m E-\frac{3}{4} k^{2} \sim m m_{\rho}$. Hence, the cusp may start interfering with the shape of resonance when $E \sim 4 m_{\rho}$; see Figs. 6-8 as an example.

\section{SUMMARY AND DISCUSSION}

In summary, we explore and experiment with an alternative approach of studying resonance properties in a finite volume. As the consequence of the global symmetry of a Green's function in the complex spatial plane, by analytic continuation of $L$ into $i L$, the oscillating behavior of a finite volume Green's function can be mapped into an infinite volume Green's function with corrections of an exponentially decaying finite volume effect. Using the finite volume size $L$ as a tuning knob, the finite volume scattering amplitude may behave similarly to an infinite volume amplitude in $i L$ space. A cusp in the three-body finite volume amplitude due to a finite volume effect is also observed; it may start interfering with and distorting the shape of a resonance while the total energy $E$ is in certain range. Nevertheless, the resonance peak is still clearly visible even in a small box. Hence, the resonance properties may be computed directly from the finite volume dynamical equations.

The analytical continuation technique presented in the paper may be useful in visualizing resonances from finite volume dynamical equations, in processes such as $\eta, \eta^{\prime} \rightarrow 3 \pi$. In order to describe a three-particle resonance, a three-body short-range interaction must be included as well. A simple model that may be used to describe a threeparticle resonance is sketched in Appendix B.

\section{ACKNOWLEDGMENTS}

We acknowledge support from the Department of Physics and Engineering, California State University, Bakersfield, CA. This research was supported in part by the National Science Foundation under Grant No. NSF PHY-1748958. P. G. acknowledges GPU computing resources (http:// complab.cs.csubak.edu) from the Department of Computer and Electrical Engineering and Computer Science at California State University-Bakersfield made available for conducting the research reported in this work. B. L. acknowledges support by the National Science Foundation of China under Grants No. 11775148 and No. 11735003.

\section{APPENDIX A: MULTIPARTICLE WAVE FUNCTION IN FINITE VOLUME}

The total multiparticle wave function is related to the total finite volume amplitude by

$$
\Phi_{L}=-G_{L}(E) T_{L}^{\mathrm{tot}}(E) .
$$

The total amplitude $T_{L}^{\text {tot }}(E)$ is usually given by the sum of multiple terms, for instance, in a three-body interaction with only a pairwise interaction; thus,

$$
T_{L}^{\mathrm{tot}}(E)=\sum_{\gamma=1}^{3} T_{L}^{(\alpha \beta)}(E), \quad \alpha \neq \beta \neq \gamma,
$$

where $T_{L}^{(\alpha \beta)}(E)$ satisfies coupled homogeneous equations, such as

$T_{L}^{(12)}(E)=\frac{1}{\frac{1}{V_{12}}-G_{L}(E)} G_{L}(E)\left[T_{L}^{(23)}(E)+T_{L}^{(31)}(E)\right]$.

For three identical particles, the coupled homogeneous equations are reduced to Eq. (36) and $T_{L}^{(\alpha \beta)}(E)=T_{L}(E)$. Therefore, finding eigensolutions of a finite volume Faddeev amplitude becomes a key step for computing the wave function.

In general, the dynamical equations of finite volume Faddeev amplitudes may be casted as a matrix equation in a linear form, 


$$
T_{i}(E)=\sum_{j} K_{i, j}(E) T_{j}(E),
$$

where the vector $T(E)$ stands for the energy dependent amplitude that also depends on the discrete momenta, and the matrix $K(E)$ represents the energy dependent kernel function. In a finite volume, due to the periodic boundary condition, the Eq. (A4) can be satisfied only for some discrete energies, $\left\{E_{1}, \ldots, E_{n}, \ldots\right\}$. Equation (A4) has nontrivial solutions only if

$$
\operatorname{det}[I-K(E)]=0, \quad E \in\left\{E_{n}\right\} .
$$

In order to find the eigenvector solution of Eq. (A4), let us consider the subtracted equation of Eq. (A4),

$T_{i}(E)=T_{i_{0}}(E)+\sum_{j}\left[K_{i, j}(E)-K_{i_{0}, j}(E)\right] T_{j}(E)$,

where $T_{i_{0}}(E)$ stands for the $i_{0}$ th element of a $T(E)$ vector we choose for the subtraction. $T_{i_{0}}(E)$ may also be used as a normalization factor and is a constant value for a fixed $E$. Hence, the solution of a subtracted Eq. (A6) is obtained by a matrix inversion,

$$
T(E)=\frac{1}{I-K(E)+K_{0}(E)} T_{0}(E)
$$

where $T_{0}(E)=T_{i_{0}}(E)$ vector is a constant for a fixed $E$, and $\left[K_{0}\right]_{i, j}(E)=K_{i_{0}, j}(E)$. The expression in Eq. (A7) is indeed the eigenvector solution of Eq. (A4) when $E \in\left\{E_{n}\right\}$, since $T\left(E_{n}\right)=K\left(E_{n}\right) T\left(E_{n}\right)$ and so is $T_{0}\left(E_{n}\right)=K_{0}\left(E_{n}\right) T\left(E_{n}\right)$. The subtracted homogeneous dynamical equations hence can be used to find eigenvector solutions of finite volume Faddeev type equations.

Next, we will just use a simple example to illustrate the above described approach. Let us consider three nonrelativistic identical bosons interacting with contact interactions in 1D. The wave function is given in terms of a finite volume Faddeev amplitude by

$$
\begin{aligned}
\phi_{L}\left(r_{13}, r_{23}\right)= & -\sum_{p_{1}, p_{2}} e^{i p_{1} r_{13}} e^{i p_{2} r_{23}} \tilde{G}_{L}\left(p_{1}, p_{2} ; E\right) \\
& \times\left[T_{L}\left(p_{1} ; E\right)+T_{L}\left(p_{2} ; E\right)+T_{L}\left(p_{3} ; E\right)\right],
\end{aligned}
$$

where $\left(p_{1}, p_{2}\right) \in \frac{2 \pi}{L} n, n \in \mathbb{Z}$, and $p_{3}=-p_{1}-p_{2} . r_{i j}$ are relative coordinates between $i$ th and $j$ th particles. The $T_{L}(E)$ satisfies the integral equation,

$$
T_{L}(k ; E)=-2 \tau_{L}^{(k)}(E) \sum_{p} L \tilde{G}_{L}(k, p ; E) T_{L}(p ; E),
$$

where $(k, p) \in \frac{2 \pi}{L} n, n \in \mathbb{Z}$. The eigenenergies are obtained by a quantization condition,

$$
\operatorname{det}\left[\delta_{k, p}-K(k, p ; E)\right]=0,
$$

where the kernel function is

$$
K(k, p ; E)=-2 \tau_{L}^{(k)}(E) L \tilde{G}_{L}(k, p ; E) .
$$

Once eigenenergies are determined, the eigenvector can be found by the matrix inversion of the subtracted Eq. (A9),

$\sum_{p}\left[\delta_{k, p}-K(k, p ; E)+K\left(k_{0}, p ; E\right)\right] T_{L}(p ; E)=T_{L}\left(k_{0} ; E\right)$,

where $k_{0}$ is the subtraction point and can be chosen arbitrarily, and $T_{L}\left(k_{0}\right)$ may be treated as a normalization factor.

\section{Multiparticle energy spectrum of a resonance model}

To make it more interesting, let us propose a resonance model with the following replacement in the contact interaction:

$$
m V_{0} \rightarrow m V_{0}+\frac{g_{\rho}}{m E-\frac{3}{4} k^{2}-m m_{\rho}},
$$

where $\left(g_{\rho}, m_{\rho}\right)$ stand for the coupling constant and mass of resonance, respectively. Therefore, the two- and threeparticle energy spectrum in the CM frame are determined by a two-body quantization condition,

$$
\frac{1}{m V_{0}+\frac{g_{\rho}}{m\left(E-m_{\rho}\right)}}-\frac{\cot \frac{\sqrt{m E}}{2} L}{2 \sqrt{m E}}=0,
$$

and a three-body quantization condition,

$$
\operatorname{det}\left[\delta_{k, p}+\frac{1}{L} \frac{2 \tau_{L}^{(\mathrm{res}, k)}(E)}{m E-k^{2}-k p-p^{2}}\right]=0,
$$

respectively, where

$$
\begin{aligned}
{\left[\tau_{L}^{(\mathrm{res}, k)}(E)\right]^{-1}=} & -\frac{1}{m V_{0}+\frac{g_{\rho}}{m E-\frac{3}{4} k^{2}-m m_{\rho}}} \\
& -i \frac{\operatorname{coth} \frac{\sqrt{m E-\frac{3}{4} k^{2}-\frac{k}{2}}}{2} L+\operatorname{coth} \frac{\sqrt{m E-\frac{3}{4} k^{2}}+\frac{k}{2}}{2} L}{4 \sqrt{m E-\frac{3}{4} k^{2}}} .
\end{aligned}
$$

The two- and three-particle energy spectra are shown in Figs. 9 and 10, respectively. The resonance shows up at the $m E_{2 b}=0.3$ location and flattens up the curves of the two-body energy spectrum as the function of $L$ near $m E_{2 b}=0.3$. A similar pattern is observed in the three-body 


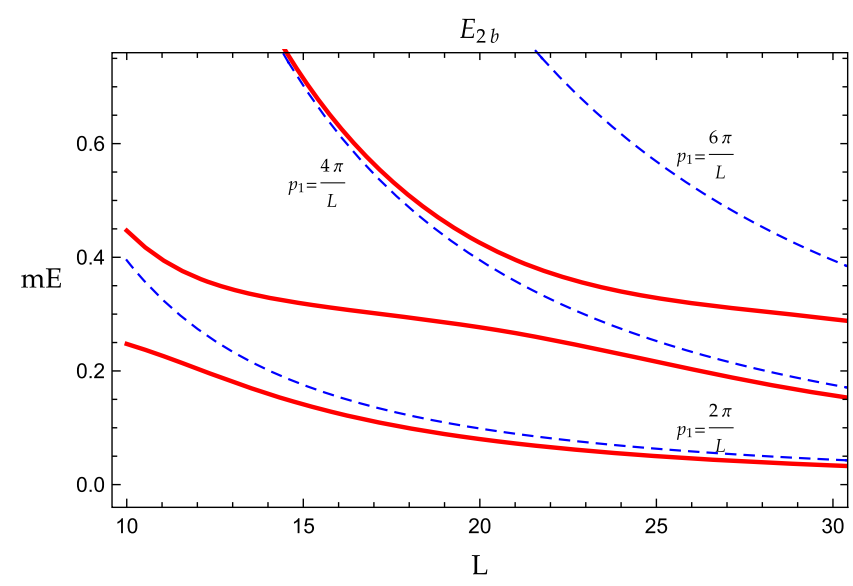

FIG. 9. The CM frame two particles energy spectrum (solid red) for a resonance model with parameters: $m V_{0}=0, g_{\rho}=0.04$, and $m m_{\rho}=0.3$. The free particles energy spectrum (dashed blue) is also plotted as a reference with $m E_{2 b}^{(\text {free })}=\left(\frac{2 \pi n}{L}\right)^{2}, n \in \mathbb{Z}$.

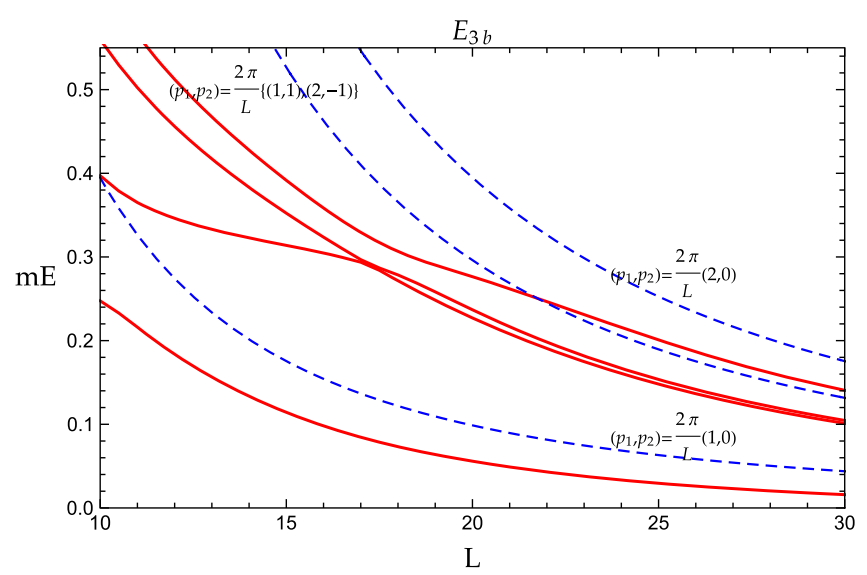

FIG. 10. The CM frame three particles energy spectrum (solid red) for a resonance model with parameters: $m V_{0}=0, g_{\rho}=0.04$, and $m m_{\rho}=0.3$. The free particles energy spectrum (dashed blue) is also plotted as a reference with $m E_{3 b}^{(\text {free })}=p_{1}^{2}+p_{1} p_{2}+p_{2}^{2}$, $\left(p_{1}, p_{2}\right) \in \frac{2 \pi n}{L}, n \in \mathbb{Z}$.

energy spectrum; however, the situation in the three-body sector is slightly more sophisticated. The energy spectrum of free particles shows the degeneracy for some levels, such as the blue curve in the middle that has a double degeneracy for $\left(p_{1}, p_{2}\right)=\frac{2 \pi}{L}(1,1)$ and $\left(p_{1}, p_{2}\right)=\frac{2 \pi}{L}(2,-1)$; hence, three blue free three-body energy spectrum curves in fact represent four energy levels. The degeneracy in the second and third levels in the middle is removed by interaction; see the splitting in the two red curves in the middle of Fig. 10.

\section{Three particles wave function of a resonance model}

With the resonance model proposed in Sec. A 1, we can thus apply the technique described previously to compute a

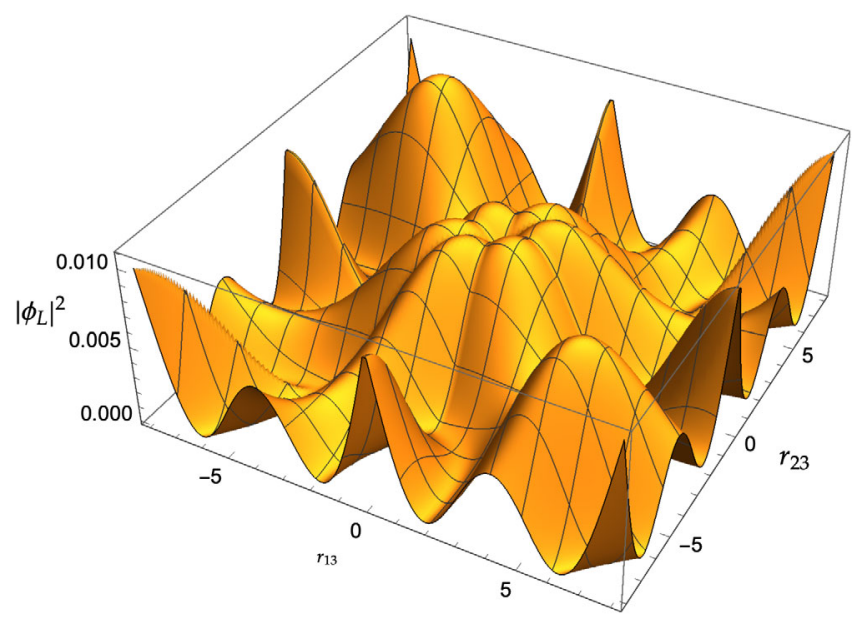

FIG. 11. Three-body wave function $\left|\phi_{L}\left(r_{13}, r_{23}\right)\right|^{2}$ for a resonance model with parameters: $m V_{0}=0, g_{\rho}=0.04, m m_{\rho}=0.3$, $L=16$, and $m E_{3 b}=0.3046 \sim m m_{\rho}$.

three particles wave function. The three-body finite volume $T_{L}$ amplitude is solved by matrix inversion and is given by

$$
T_{L}(p ; E)=\sum_{k}\left[D^{-1}\left(k_{0}, E\right)\right]_{p, k} T_{L}\left(k_{0} ; E\right),
$$

where $k_{0}$ is an arbitrary subtraction point, and the $D$ matrix is given by

$$
D_{k, p}\left(k_{0} ; E\right)=\delta_{k, p}-K(k, p ; E)+K\left(k_{0}, p ; E\right) .
$$

Using $T_{L}(p ; E)$ as input, the three-body wave function can be computed by Eq. (A8); see Fig. 11 as an example with $m E_{3 b}$ as a near resonance mass.

\section{APPENDIX B: THREE-PARTICLE RESONANCE MODEL}

In order to describe a three-particle resonance decay or scattering process, the three-body short-range interaction must be included. In this section, we present a simple threebody contact interaction model that may be useful to describe a process such as $\eta \rightarrow 3 \pi$. When the three-body short-range interaction is included, all the discussion presented in Sec. II D must be extended by including a three-body amplitude $T^{(3 b)}$. Thus, Eq. (38) is replaced by coupled equations,

$\mathcal{T}_{L}(E)=\tau_{L}(E)-\tau_{L}(E) G_{L}(E)\left[2 \mathcal{T}_{L}(E)+\mathcal{T}_{L}^{(3 b)}(E)\right]$,

and

$$
\mathcal{T}_{L}^{(3 b)}(E)=\tau_{L}^{(3 b)}(E)-\tau_{L}^{(3 b)}(E) G_{L}(E) 3 \mathcal{T}_{L}(E),
$$

where $\tau_{L}^{(3 b)}$ is related to the three-body interaction $V_{123}$ by 


$$
\tau_{L}^{(3 b)}(E)=-\frac{1}{\frac{1}{V_{123}}-G_{L}(E)}
$$

With the same convention, $\tau_{L}$ is related to the two-body pairwise interaction $V$ by

$$
\tau_{L}(E)=-\frac{1}{\frac{1}{V}-G_{L}(E)} .
$$

Eliminating $\mathcal{T}_{L}^{(3 b)}$ in Eq. (B1), we thus find

$$
\begin{aligned}
\mathcal{T}_{L}(E)= & \tau_{L}(E)\left[1-G_{L}(E) \tau_{L}^{(3 b)}(E)\right] \\
& -\tau_{L}(E) G_{L}(E)\left[2-3 \tau_{L}^{(3 b)}(E) G_{L}(E)\right] \mathcal{T}_{L}(E)
\end{aligned}
$$

The quantization condition Eq. (37) is thus replaced by $\operatorname{det}\left[I+\tau_{L}(E) G_{L}(E)\left(2-3 \tau_{L}^{(3 b)}(E) G_{L}(E)\right)\right]=0$,

where the three-body term $\tau_{L}^{(3 b)}(E)$ may be modeled to describe the impact of the three-body resonance to the spectrum of a three-body system.

In a momentum basis with a short-range three-body interaction, Eq. (B5) is thus given by

$$
\begin{aligned}
\mathcal{T}_{L}(k ; E)= & \tau_{L}^{(k)}(E)\left[1-\left(\sum_{p^{\prime}} L \tilde{G}_{L}\left(k, p^{\prime} ; E\right)\right) \tau_{L}^{(3 b)}(E)\right] \\
& -\tau_{L}^{(k)}(E) \sum_{p}\left[2 L \tilde{G}_{L}(k, p ; E)\right. \\
& -3\left(\sum_{p^{\prime}} L \tilde{G}_{L}\left(k, p^{\prime} ; E\right)\right) \\
& \left.\times \tau_{L}^{(3 b)}(E)\left(\sum_{p^{\prime \prime}} L \tilde{G}_{L}\left(p^{\prime \prime}, p ; E\right)\right)\right] \mathcal{T}_{L}(p ; E),
\end{aligned}
$$

where

$$
\tau_{L}^{(3 b)}(E)=-\frac{1}{\frac{1}{m V_{123}}-\sum_{k, p} \tilde{G}_{L}(k, p ; E)} .
$$

The three-particle resonance may be modeled by replacing $V_{123}$ by

$$
m V_{123}+\frac{g_{123}}{m\left(E-m_{123}\right)},
$$

where $g_{123}$ and $m_{123}$ represent the coupling strength and mass of three-body resonance.
[1] J. Kambor, C. Wiesendanger, and D. Wyler, Nucl. Phys. B465, 215 (1996).

[2] A. V. Anisovich and H. Leutwyler, Phys. Lett. B 375, 335 (1996).

[3] S. P. Schneider, B. Kubis, and C. Ditsche, J. High Energy Phys. 02 (2011) 028.

[4] K. Kampf, M. Knecht, J. Novotny, and M. Zdrahal, Phys. Rev. D 84, 114015 (2011).

[5] P. Guo, I. V. Danilkin, D. Schott, C. Fernández-Ramírez, V. Mathieu, and A. P. Szczepaniak, Phys. Rev. D 92, 054016 (2015).

[6] P. Guo, I. V. Danilkin, C. Fernández-Ramírez, V. Mathieu, and A. P. Szczepaniak, Phys. Lett. B 771, 497 (2017).

[7] G. Colangelo, S. Lanz, H. Leutwyler, and E. Passemar, Phys. Rev. Lett. 118, 022001 (2017).

[8] P. Guo, R. Mitchell, and A. P. Szczepaniak, Phys. Rev. D 82, 094002 (2010).

[9] P. Guo, R. Mitchell, M. Shepherd, and A. P. Szczepaniak, Phys. Rev. D 85, 056003 (2012).

[10] S. Aoki et al. (CP-PACS Collaboration), Phys. Rev. D 76, 094506 (2007).

[11] X. Feng, K. Jansen, and D. B. Renner, Phys. Rev. D 83, 094505 (2011).

[12] C. B. Lang, D. Mohler, S. Prelovsek, and M. Vidmar, Phys. Rev. D 84, 054503 (2011); 89, 059903(E) (2014).
[13] S. Aoki et al. (CS Collaboration), Phys. Rev. D 84, 094505 (2011).

[14] J. J. Dudek, R. G. Edwards, and C. E. Thomas, Phys. Rev. D 86, 034031 (2012).

[15] J. J. Dudek, R. G. Edwards, and C. E. Thomas (Hadron Spectrum Collaboration), Phys. Rev. D 87, 034505 (2013); 90, 099902(E) (2014).

[16] D. J. Wilson, J. J. Dudek, R. G. Edwards, and C. E. Thomas, Phys. Rev. D 91, 054008 (2015).

[17] D. J. Wilson, R. A. Briceño, J. J. Dudek, R. G. Edwards, and C. E. Thomas, Phys. Rev. D 92, 094502 (2015).

[18] J. J. Dudek, R. G. Edwards, and D. J. Wilson (Hadron Spectrum Collaboration), Phys. Rev. D 93, 094506 (2016).

[19] S. R. Beane, W. Detmold, T. C. Luu, K. Orginos, M. J. Savage, and A. Torok, Phys. Rev. Lett. 100, 082004 (2008).

[20] W. Detmold, M. J. Savage, A. Torok, S. R. Beane, T. C. Luu, K. Orginos, and A. Parreno, Phys. Rev. D 78, 014507 (2008).

[21] B. Hörz and A. Hanlon, Phys. Rev. Lett. 123, 142002 (2019).

[22] C. Andersen, J. Bulava, B. Hörz, and C. Morningstar, Nucl. Phys. B939, 145 (2019).

[23] B. Hörz et al., arXiv:2009.11825.

[24] R. Brett, J. Bulava, J. Fallica, A. Hanlon, B. Hörz, and C. Morningstar, Nucl. Phys. B932, 29 (2018). 
[25] C. Alexandrou, L. Leskovec, S. Meinel, J. Negele, S. Paul, M. Petschlies, A. Pochinsky, G. Rendon, and S. Syritsyn, Phys. Rev. D 96, 034525 (2017).

[26] M. Fischer, B. Kostrzewa, J. Ostmeyer, K. Ottnad, M. Ueding, and C. Urbach, Eur. Phys. J. A 56, 206 (2020).

[27] C. W. Andersen, J. Bulava, B. Hörz, and C. Morningstar, Phys. Rev. D 97, 014506 (2018).

[28] M. Lüscher, Nucl. Phys. B354, 531 (1991).

[29] K. Rummukainen and S. A. Gottlieb, Nucl. Phys. B450, 397 (1995).

[30] N. H. Christ, C. Kim, and T. Yamazaki, Phys. Rev. D 72, 114506 (2005).

[31] V. Bernard, M. Lage, U.-G. Meißner, and A. Rusetsky, J. High Energy Phys. 08 (2008) 024.

[32] S. He, X. Feng, and C. Liu, J. High Energy Phys. 07 (2005) 011.

[33] M. Lage, U.-G. Meißner, and A. Rusetsky, Phys. Lett. B 681, 439 (2009).

[34] M. Döring, U.-G. Meißner, E. Oset, and A. Rusetsky, Eur. Phys. J. A 47, 139 (2011).

[35] R. A. Briceño and Z. Davoudi, Phys. Rev. D 88, 094507 (2013).

[36] M. T. Hansen and S. R. Sharpe, Phys. Rev. D 86, 016007 (2012).

[37] P. Guo, J. Dudek, R. Edwards, and A. P. Szczepaniak, Phys. Rev. D 88, 014501 (2013).

[38] P. Guo, Phys. Rev. D 88, 014507 (2013).

[39] D. Agadjanov, M. Döring, M. Mai, U.-G. Meißner, and A. Rusetsky, J. High Energy Phys. 06 (2016) 043.

[40] S. Kreuzer and H. W. Hammer, Phys. Lett. B 673, 260 (2009).

[41] S. Kreuzer and H. W. Hammer, Eur. Phys. J. A 43, 229 (2010).

[42] S. Kreuzer and H. W. Grießhammer, Eur. Phys. J. A 48, 93 (2012).

[43] K. Polejaeva and A. Rusetsky, Eur. Phys. J. A 48, 67 (2012).

[44] R. A. Briceño and Z. Davoudi, Phys. Rev. D 87, 094507 (2013).

[45] M. T. Hansen and S. R. Sharpe, Phys. Rev. D 90, 116003 (2014).

[46] M. T. Hansen and S. R. Sharpe, Phys. Rev. D 92, 114509 (2015).

[47] M. T. Hansen and S. R. Sharpe, Phys. Rev. D 93, 096006 (2016); 96, 039901(E) (2017).

[48] R. A. Briceño, M. T. Hansen, and S. R. Sharpe, Phys. Rev. D 95, 074510 (2017).
[49] S. R. Sharpe, Phys. Rev. D 96, 054515 (2017); 98, 099901 (E) (2018).

[50] H.-W. Hammer, J.-Y. Pang, and A. Rusetsky, J. High Energy Phys. 09 (2017) 109.

[51] H. W. Hammer, J. Y. Pang, and A. Rusetsky, J. High Energy Phys. 10 (2017) 115.

[52] U.-G. Meißner, G. Ríos, and A. Rusetsky, Phys. Rev. Lett. 114, 091602 (2015); 117, 069902(E) (2016).

[53] M. Mai and M. Döring, Eur. Phys. J. A 53, 240 (2017).

[54] M. Mai and M. Döring, Phys. Rev. Lett. 122, 062503 (2019).

[55] M. Döring, H. W. Hammer, M. Mai, J. Y. Pang, A. Rusetsky, and J. Wu, Phys. Rev. D 97, 114508 (2018).

[56] F. Romero-López, A. Rusetsky, and C. Urbach, Eur. Phys. J. C 78, 846 (2018).

[57] P. Guo, Phys. Rev. D 95, 054508 (2017).

[58] P. Guo and V. Gasparian, Phys. Lett. B 774, 441 (2017).

[59] P. Guo and V. Gasparian, Phys. Rev. D 97, 014504 (2018).

[60] P. Guo and T. Morris, Phys. Rev. D 99, 014501 (2019).

[61] T. D. Blanton, F. Romero-López, and S. R. Sharpe, J. High Energy Phys. 03 (2019) 106.

[62] F. Romero-López, S. R. Sharpe, T. D. Blanton, R. A. Briceño, and M. T. Hansen, J. High Energy Phys. 10 (2019) 007.

[63] T. D. Blanton, F. Romero-López, and S. R. Sharpe, Phys. Rev. Lett. 124, 032001 (2020).

[64] M. Mai, M. Döring, C. Culver, and A. Alexandru, Phys. Rev. D 101, 054510 (2020).

[65] P. Guo, M. Döring, and A. P. Szczepaniak, Phys. Rev. D 98, 094502 (2018).

[66] P. Guo, Phys. Lett. B 804, 135370 (2020).

[67] P. Guo and M. Döring, Phys. Rev. D 101, 034501 (2020).

[68] P. Guo, Phys. Rev. D 101, 054512 (2020).

[69] P. Guo and B. Long, Phys. Rev. D 101, 094510 (2020).

[70] P. Klos, S. König, H. Hammer, J. Lynn, and A. Schwenk, Phys. Rev. C 98, 034004 (2018).

[71] P. Guo, arXiv:2007.04473.

[72] M. Döring, M. Mai, and U.-G. Meißner, Phys. Lett. B 722, 185 (2013).

[73] Y. Ho, Phys. Rep. 99, 1 (1983).

[74] N. Moiseyev, Phys. Rep. 302, 212 (1998).

[75] N. Moiseyev, Non-Hermitian Quantum Mechanics (Cambridge University Press, Cambridge, England, 2011).

[76] P. Guo, I. V. Danilkin, and A. P. Szczepaniak, Eur. Phys. J. A 51, 135 (2015). 